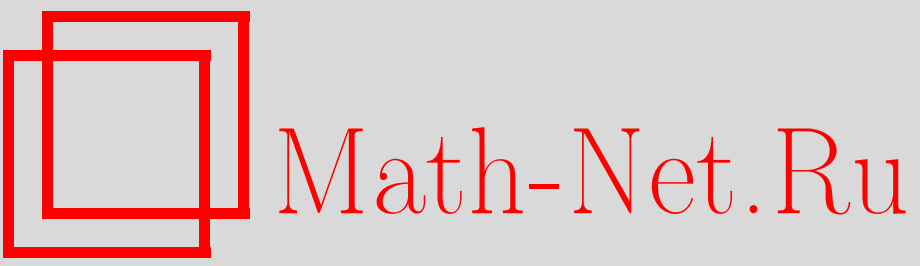

Е. П. Мочалина, Об одном критерии аналитической продолжимости функции C отрезка, УМH, 2003, том 58, выпуск 6, 161-162

DOI: https://doi.org/10.4213/rm687

Использование Общероссийского математического портала Math-Net.Ru подразумевает, что вы прочитали и согласны с пользовательским соглашением

http://www.mathnet.ru/rus/agreement

Параметры загрузки:

IP: 54.210 .77 .194

26 апреля 2023 г., 17:15:36 


\title{
ОБ ОДНОМ КРИТЕРИИ АНАЛИТИЧЕСКОЙ ПРОДОЛЖИМОСТИ ФУНКЦИИ $C$ ОТРЕЗКА
}

\author{
Е. П. МочАлинА
}

В анализе хорошо известен результат С. Н. Бернштейна [1]: условие $\limsup _{n \rightarrow \infty} \sqrt[n]{E_{n}(f)}=$ $\rho<1, E_{n}(f)=\inf _{s \in p_{n}}\|f-s\|_{C(\Delta)}$, а $f$ непрерывна на $\Delta=[-1 ; 1]$, равносильно тому, что существует аналитическое продолжение в область $\Gamma_{\rho}$, ограниченную эллипсом с фокусами \pm 1 и суммой полуосей $1 / \rho$, имеющее хотя бы одну особую точку на границе $\partial \Gamma \rho$. Для заданной функции $f \in L_{p}(\Delta)$ определим величины наименьших уклонений в $L_{p}(\Delta)$ от $p_{n}=\left\{\sum_{j=0}^{k} c_{j} x^{j}\right.$, $\left.k \leqslant n, c_{j} \in \mathbb{C}\right\}$ и $r_{n, n}=\left\{g / s: g, s \in p_{n}\right\}: E_{n}=\inf _{s \in p_{n}}\|f-s\|, R_{n} \equiv R_{n}(f)=$ $\inf _{r \in r_{n, n}}\|f-r\|$.

Теорема. Пусть фиксированы параметр $p \in(1 ;+\infty)$ u $f \in L_{p}[-1 ; 1]$. Условие $\limsup _{n \rightarrow \infty} \sqrt[n]{E_{n}-R_{n+1}}=\rho<1$ необходимо и достаточно для того, чтобь $f$ аналитически продолжалась в область Г и н на Г имела хотя бы одну особую точку.

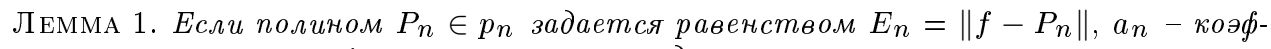
фичиент при его старшей степени, то справедливо неравенство:

$$
E_{n}-E_{n+1} \leqslant 2\left(1+\left|a_{n+1} / a_{n}\right|\right) \sqrt{E_{n}\left(E_{n}-R_{n+1}\right)} .
$$

ДокАЗАТЕЛЬСтво. Пусть $m=n+1-\operatorname{deg} P_{n}, \gamma=a_{n} / a_{n+1}, T=\left(\left(1+\gamma-\lambda x^{m}\right) P_{n}+\right.$ $\left.\lambda \gamma P_{n+1}\right) /\left(1-\lambda x^{m}\right)$, где $\lambda \in \mathbb{C}, \lambda \neq \lambda(x),|\lambda| \leqslant 1 / 2$. Так как $T \in r_{n+1, n+1}$ и $T-f=(1-$ $\lambda \gamma)\left(P_{n}-f\right)+\lambda \gamma\left(P_{n+1}-f\right)+\lambda^{2} \gamma\left(P_{n+1}-P_{n}\right) x^{m} /\left(1-\lambda x^{m}\right)$, то $R_{n+1} \leqslant|1-\lambda \gamma| E_{n}+$ $|\lambda \gamma| E_{n+1}+4\left|\lambda^{2} \gamma\right| E_{n}$. Выберем $\lambda$ из условия $|1-\lambda \gamma|=1-|\lambda \gamma|$ и перепишем последнее неравенство: $E_{n}-E_{n+1} \leqslant\left(E_{n}-R_{n+1}\right) /|\lambda \gamma|+4|\lambda| E_{n}$. Положив $2 \lambda=\sqrt{\left(E_{n}-R_{n+1}\right) / E_{n}}$. $\exp (-i \arg \gamma),|\gamma| \leqslant 1,2 \lambda \gamma=\sqrt{\left(E_{n}-R_{n+1}\right) / E_{n}},|\gamma|>1$. Завершим доказательство леммы.

Пусть $L_{k}(x)=\mu_{k} x^{k}+\cdots-$ ортонормированньй многочлен Лежандра степени $k, q=$ $p /(p-1)$. Далее для сокращения записи постоянные, не зависящие от $n$, обозначаем $C$.

Лемма 2. Если $E_{n}=\left\|f-P_{n}\right\|, \quad i_{n}=\operatorname{deg} P_{n}, \quad P_{n}(x)=a_{n} x^{i_{n}}+\cdots, \quad \tilde{f}=f-$ $\left(a_{n} / \mu_{i_{n+1}}\right) L_{i_{n}}, \Theta_{n}=R_{n}(\widetilde{f})$, mo

$$
E_{n}-E_{n+1} \leqslant C\left\{\begin{array}{l}
E_{n}^{1 / 2}\left(E_{n}-\Theta_{n+1}\right)^{1 / 2}, \quad p \geqslant 2, \\
E_{n}^{1 / p}\left(E_{n}-\Theta_{n+1}\right)^{1 / q}, \quad 1<p<2 .
\end{array}\right.
$$

ДокаЗАТЕЛЬСтво. Считаем, что $\operatorname{deg} P_{n+1}=n+1$ (иначе $E_{n}=E_{n+1}$ ). Пусть $\lambda, b \in \mathbb{C}$, $\lambda \neq \lambda(x), b \neq b(x),|b|=2 ; F_{n+1}=P_{n}-\left(a_{n} / \mu_{i_{n+1}}\right) L_{i_{n}}-\lambda b\left(P_{n+1}-P_{n}\right) /(x-b)$. Тогда

$$
\Theta_{n+1}^{p} \leqslant\left\|\tilde{f}-F_{n+1}\right\|^{p}=\left\|f-P_{n}+\lambda b\left(P_{n+1}-P_{n}\right) /(x-b)\right\|^{p} .
$$

При $\alpha \in \mathbb{C}$ положим $V(t)=|\alpha+t|^{p}, t \in \Delta$. Пусть $p \geqslant 2$. Тогда по формуле Тейлора имеем $V(t)-V(0)-t V^{\prime}(0)=t^{2} V^{\prime \prime}(\zeta t) / 2 \leqslant C t^{2}\left(|\alpha|^{p-2}+|t|^{p-2}\right)$. Если $\alpha=\psi / \beta$, то отсюда следует

$$
|\psi+t \beta|^{p} \leqslant|\psi|^{p}+p t|\psi|^{p-2} \operatorname{Re}(\bar{\psi} \beta)+C t^{2}|\beta|^{2}\left(|\psi|^{p-2}+|\beta|^{p-2}\right) .
$$

Полагаем $\phi_{n}=\left|f-P_{n}\right|^{p-2} \overline{\left(f-P_{n}\right)}, \sigma=\int_{\Delta} \phi_{n}\left(P_{n+1}-P_{n}\right) d x$. Тогда $|\sigma|=\mid E_{n}^{p}-\int_{\Delta} \phi_{n}(f-$ $\left.P_{n+1}\right) d x \mid \geqslant\left(E_{n}-E_{n+1}\right) E_{n}^{p-1}$. Если $\sigma=0$, то $E_{n}=E_{n+1}$ и, следовательно, справедли-

Работа выполнена по гранту Государственной поддержки ведущих научных школ № НШ1892.2003.1. 
вость (2). Поэтому считаем $\sigma \neq 0$ и полагаем в (4) $\psi=f-P_{n}, \beta=\lambda b\left(P_{n+1}-P_{n}\right) /(x-b)$ при $\lambda=-|\sigma| / \sigma$ и интегрируем (4) по $\Delta$. Используя неравенства (3), $\int_{\Delta}|\psi(x)|^{p-2} d x \leqslant 2\|\psi\|^{p-2}$, $\|\beta\| \leqslant C E_{n}$ и $\|\psi\|=E_{n}$, имеем

$$
\Theta_{n+1}^{p} \leqslant E_{n}^{p}+t p \operatorname{Re}\left(\int_{\Delta}|\psi|^{p-2} \bar{\psi} \beta d x\right)+C t^{2} E_{n}^{p}=E_{n}^{p}-t p|\sigma|+t p \operatorname{Re}(A(b))+C t^{2} E_{n}^{p},
$$

где $A(b)=-|\sigma| / \sigma \int_{\Delta} x \phi_{n}\left(P_{n+1}-P_{n}\right) /(x-b) d x$. Покажем, что на границе $\partial U$ круга $U=$ $\{b:|b| \leqslant 2\}$ существует хотя бы одна точка $b$ такая, что $A(b)<0$. Функция $A(b)$ голоморфона на $\overline{\mathbb{C}} \backslash\{z:|z| \leqslant 1\}$. Пусть $G$ - образ внешности круга $U$ при отображении $\nu=A(b)$. Так как $A(\infty)=0$, то $G$ - ограниченная область и $0 \in G$. Граница области $G$ пересекает отрицательную полуось действительной оси $\operatorname{Im} \nu=0$ в точке $\nu_{0}=\sup \{\nu: \nu<0, \nu \notin G\}$. Выбрав $b=\nu^{-1}\left(\nu_{0}\right)$, имеем $A(b)<0$. Теперь из (5) и (1) следует $E_{n}^{p}-\Theta_{n+1}^{p} \geqslant t E_{n}^{p-1}\left(E_{n}-E_{n+1}\right)-t^{2} C E_{n}^{p}$. Выбрав $t=\left(E_{n}-E_{n+1}\right) /\left(E_{n} C\right)$ и заметив, что $x^{p}-y^{p} \leqslant p x^{p-1}$, получим (2) для $p \geqslant 2$.

Рассмотрим $p \in(1 ; 2)$. По теореме Лагранжа $V(t)-V(0)-t V^{\prime}(0)=t\left(V^{\prime}(\zeta t)-V^{\prime}(0)\right)$. При $t>0$ оценим сверху $N=\left|V^{\prime}(\zeta t)-V^{\prime}(0)\right|$. Если $|\alpha| \leqslant t$, то, с учетом $|a+c|^{p-1} \leqslant|a|^{p-1}+|c|^{p-1}$, $a, c \in \mathbb{C}$, получим $N \leqslant 6 t^{p-1}$. Пусть $|\alpha|>t$. Используя $\left.|| a\right|^{p-1}-|c|^{p-1}|\leqslant| a+\left.c\right|^{p-1}$, найдем, что $N \leqslant p\left(2 t /|\alpha|^{2-p}+3 t^{p-1}\right)<C t^{p-1}$. Теперь из вида $V(t)$ при $\alpha=\psi / \beta$ получаем аналог (4): $|\psi+t \beta|^{p} \leqslant|\psi|^{p}+t p|\psi|^{p-2} \operatorname{Re}(\bar{\psi} \beta)+C t^{p}|\beta|^{p}$. Далее, рассуждая, как и при $p \geqslant 2$ имеем $E_{n}^{p}-\Theta_{n+1}^{p} \geqslant t p E_{n}^{p-1}\left(E_{n}-E_{n+1}\right)-C t^{p} E_{n}^{p}$, что при $t=\left(\left(E_{n}-E_{n+1}\right) / E_{n}\right)^{1 /(p-1)}$ доказьвает лемму.

Лемма 3. Пусть $E_{n}-R_{n+1}=O\left(\rho^{n}\right)$. Тогда $E_{n}=O\left(n^{2(\nu+1)} \rho^{n}\right), \nu=\max \{2, q\}$.

ДокАЗАТЕЛЬСТвО. Пусть $1<p \leqslant 2, \chi_{n+1} \in r_{n+1, n+1}: \Theta_{n+1}=\left\|\widetilde{f}-\chi_{n+1}\right\|$. Имеем $R_{n+1} \leqslant\left\|f-\chi_{n+1}\right\|=\left\|\widetilde{f}-\chi_{n+1}+\left(a_{i_{n}} / \mu_{i_{n+1}}\right) L_{i_{n}}\right\| \leqslant \Theta_{n+1}+2 n\left|a_{i_{n}}\right| / \mu_{i_{n+1}}, E_{n}-\Theta_{n+1} \leqslant$ $E_{n}-R_{n+1}+2 n\left|a_{i_{n}}\right| / \mu_{i_{n+1}}$. Считаем $\alpha=1-\beta, \beta=q /(2 q+2)$. Предположим, что $\left|a_{n}\right| \leqslant$ $E_{n}^{\alpha}\left(E_{n}-R_{n+1}\right)^{\beta} \mu_{i_{n+1}}$. Тогда из последнего неравенства и предположения имеем $E_{n}-\Theta_{n+1} \leqslant$ $E_{n}-R_{n+1}+2 n E_{n}^{\alpha}\left(E_{n}-R_{n+1}\right)^{\beta} \leqslant 3 n E_{n}^{\alpha}\left(E_{n}-R_{n+1}\right)^{\beta}$. Из леммы 2 и равенства $q / p+$ $\alpha=q(2 q+1) /(2 q+2) \equiv q(1-\gamma)$ находим $E_{n}-E_{n+1} \leqslant C E_{n}^{1-\gamma}\left(E_{n}-R_{n+1}\right)^{\gamma}$. Пусть $\left|a_{n}\right| \geqslant$ $E_{n}^{\alpha}\left(E_{n}-R_{n+1}\right)^{\beta} \mu_{i_{n+1}}$. Заметим, что $\left|a_{n+1}\right| \leqslant 16 n \mu_{i_{n+1}} E_{n}$ в силу ортонормированности $L_{k}$ и неравенства Гёльдера. Поэтому $\left|a_{n+1} / a_{n}\right| \leqslant 16 n\left(E_{n} /\left(E_{n}-R_{n+1}\right)\right)^{\beta}$. Отсюда и из (1) имеем $E_{n}-E_{n+1} \leqslant C n E_{n}^{1-\gamma}\left(E_{n}-R_{n+1}\right)^{\gamma}$. Из леммы 2 легко получаем такие же неравенства при $p>2$. Положим $\nu=\max \{2, q\}$. Так как $E_{n}-R_{n+1}=O\left(\rho^{n}\right)$, то $E_{n}=\sum_{m=n}^{\infty}\left(E_{m}-E_{m+1}\right) \leqslant$ $C E_{n}^{1-\gamma} \rho^{n \gamma} \sum_{m=n}^{\infty} m \rho^{(m-n) \gamma} \leqslant C n^{2} E_{n}^{1-\gamma} \rho^{n \gamma}$, а значит $E_{n}=O\left(n^{2(\nu+1)} \rho^{n}\right)$.

По неравенству Никольского (см. [2]) $\left\|Q_{n}\right\|_{C(\Delta)} \leqslant C n^{2 / p}\left\|Q_{n}\right\|$, поэтому из условия теоремы и леммы 3 следует $\left\|P_{n+1}-P_{n}\right\|_{C(\Delta)} \leqslant C n^{2 / p}\left\|P_{n}-P_{n+1}\right\| \leqslant n^{4(\nu+1)+2 / p} \rho^{n}$. Далее, $f=P_{1}+\sum_{s=1}^{\infty}\left(P_{s+1}-P_{s}\right)$. Поскольку $\left|Q_{n}(z)\right|<M R^{n}$ на $\Gamma_{1 / R}$, если только $\left|Q_{n}(x)\right|<M$ на $\Delta$, то на $\Gamma_{\rho+\varepsilon}\left|P_{s+1}-P_{s}\right|<C(\rho /(\rho+\varepsilon))^{n}$, а значит, ряд равномерно сходится внутри всякого эллипса, сумма полуосей которого менее $1 / \rho$, и тем самым представляет там аналитическую функцию. Доказательство теоремы заканчивается применением приведенного выше резултата С. Н. Бернштейна.

\section{СПИСОК ЛИТЕРАТУРЫ}

[1] С.Н.Бернштейн. Экстремальные свойства полиномов и наилучшее приближение непрерывных функций одной вещественной переменной. М.-Л.: ОНТИ, 1937. [2] С. М. Никольский. Неравенства для целых функций конечной степени и их применение в теории дифференцируемых функций многих переменных // Труды МИАН. 1951. Т. 217. С. 135-181.

Российский государственный технологический университет им. К.Э. Циолковского
Принято редколлегией 23.09.2003 